\title{
Use of artificial intelligence for management and identification of complications in diabetes
}

\section{ABSTRACT}

Artificial intelligence (Al) can play an important role is in early diagnosis of complications, adherence to a healthy lifestyle and medication, real-time monitoring for optimal glycemic status and predictive prognostic model for the diabetic status of a patient. The early recognition and management of the complications (acute as well as chronic) in diabetes predict the quality of life (QoL) of a patient. The promising results of $\mathrm{Al}$ in the early diagnosis of diabetic retinopathy have opened the frontiers for management of other complications as well. Although flash glucose monitoring (FGMs) and continuous glucose monitoring(CGMs) are yet to be used in routine clinical practice but these modalities do hold promise in future for management of diabetes. Automated diagnosis of diabetic retinopathy (DR) and cardiovascular risk factor monitoring are now possible based on the large retinal fundus imaging datasets with improved sensitivity and specificity. Smart-phones and smart devices do have the potential to bring the monitoring and early diagnosis of complications of diabetes into the patient's domain with the use of applications on their smart devices which will make future management of diabetes as an "e-disease management". Al applications offer greater accuracy, efficiency, ease of use and satisfaction and can com-

Address for correspondence:

Ashish Behera

Department of Internal Medicine,

Postgraduate Institute of Medical Education and Research,

Chandigarh, India

e-mail: drashishbehera@gmail.com

Clinical Diabetology 2021, 10; 2: 221-225

DOI: $10.5603 /$ DK.a2021.0007

Received: 29.09.2020

Accepted: 28.12 .2020 plement the management and early identification of complication of diabetes in long run. (Clin Diabetol 2021; 10; 2: 221-225)

Key words: artificial intelligence, fuzzy logic, diabetic retinopathy, diabetes monitoring devices, machine learning

\section{Introduction}

"Intelligence" is a combination of various abilities which made humans stand ahead in the animal kingdom, defined by Cambridge dictionary; "is the ability to learn, understand and make judgments or have opinions that are based on reason" [1]. The new aim to impart the computers with intelligence opened frontiers of "artificial intelligence (Al)" and has been aptly defined by Boden in 1977, "is the ability to make computers do things that would require intelligence if done by humans" [2]. Al methodologies and techniques which include case-based reasoning, machine learning and deep learning [artificial neural networks (ANNs), support vector machine, naïve Bayes, decision trees, random forest, classification and regression trees, and k-nearest neighbor], evolutionary computations and hybrid intelligent systems and fuzzy logic (FL) are being applied to medicine and health from last few decades. These methods are helpful for disease diagnosis, classification and assisting the health care professionals for tasks which require manipulation of data and knowledge $[3,4]$. Use of smart phones with improved cameras and biometry as well as fitness wearable devices with faster computational capabilities has opened up new frontiers for heath industry. In 2017 in a survey, $68 \%$ of mobile health app developers believed 
Table 1. Overview of achievements in diabetology with use of digital technology and artificial intelligence

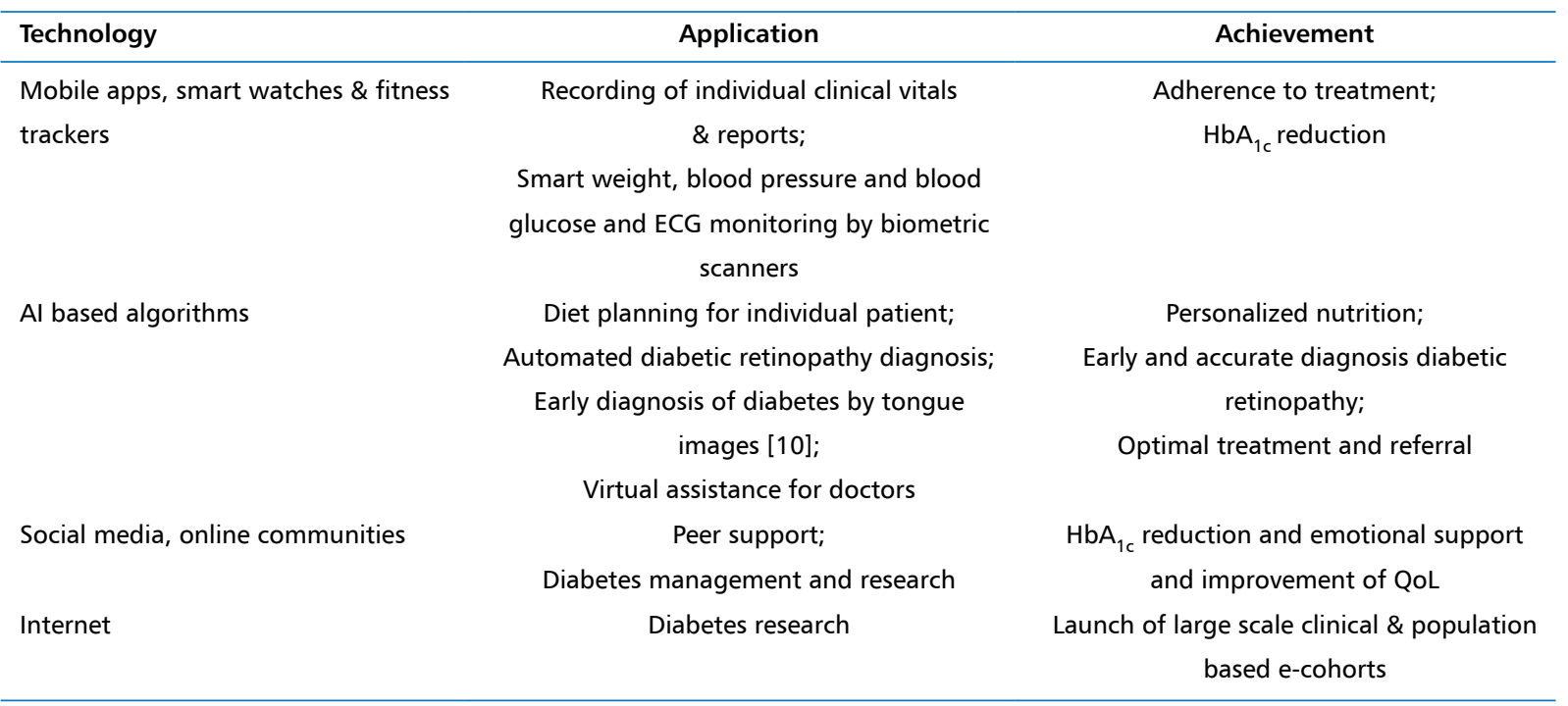

diabetes continues to be the single disease with the best market potential for digital health solutions and $61 \%$ saw $\mathrm{Al}$ as an disruptive technology for diabetes management [5].

With a staggering population of 463 million people with diabetes in 2019 which is predicted to increase to a staggering figure of 700 million in 2045 and about accounting up to $10 \%$ of the global health expenditure, diabetes is a prime disease that can benefit from use of Al and cognitive computing $[5,6]$. The challenges for which Al can play an important role is in early diagnosis of complications, adherence to a healthy lifestyle and medication, real-time monitoring for optimal glycemic status and predictive prognostic model for the diabetic status of a patient.

\section{Role of Al in diabetes}

Management of diabetes mellitus in a patient is not mere control of $\mathrm{HbA} 1 \mathrm{c}$ but requires a timely diagnosis, education of the patient in self management and continuous medical care to prevent the acute (hypoglycaemia, keto-acidosis etc.) and early recognition for reduction of long term complications (e.g. neuropathy, retinopathy, nephropathy, diabetic foot, stroke, cardiovascular and peripheral vascular diseases) for improvement of the QoL. Patient decision-support aids have been used for various purposes for decision making in people with diabetes about options like the treatment choices or setting the therapeutic goals and have been proven in improving the quality of decision making and increase of knowledge transfer [7]. The amount of data each person living with diabetes can generate is huge and can amount to 1 million gigabytes but most of the data is unstructured [5]. Rapid advances in Al offer promise for making both the real time structured and unstructured data available for person living with diabetes. The digital technologies used by individuals generate a large data set which constitute a 'digitosome', which when combined with the clinical data can be very helpful for digital algorithm and patterns [8].

The use of Al for efficient data handling and the development of tools and devices for management of diabetes are being explored and hold promise in recent future. Introduction of wearable devices, smart-phones influences and improves the cohesive integrity for the benefit of the patients with diabetes, health care professionals and the health care system in a comprehensive way. The various dimensions being explored for use of digital technologies in diabetology include use of mobile apps, Al-based algorithms, continuous/ flash glucose monitoring (CGM), closed loop systems (artificial pancreas), social media and on-line communities (Table 1). In comparison to other chronic diseases, with diabetes self monitoring is an integral part and can generate substantial data and biomarkers for e-health tools for tracking patterns and can used to correlate with the lifestyle modification as well as planning the interventional approach [9].

\section{How does Al work?}

Understanding Al or computational intelligence may be beyond the scope of this article but understanding the principle of methodologies is quite basic (Figure 1). The various techniques implemented include:

- case-based reasoning (CBR);

- machine learning and deep learning;

- miscellaneous. 


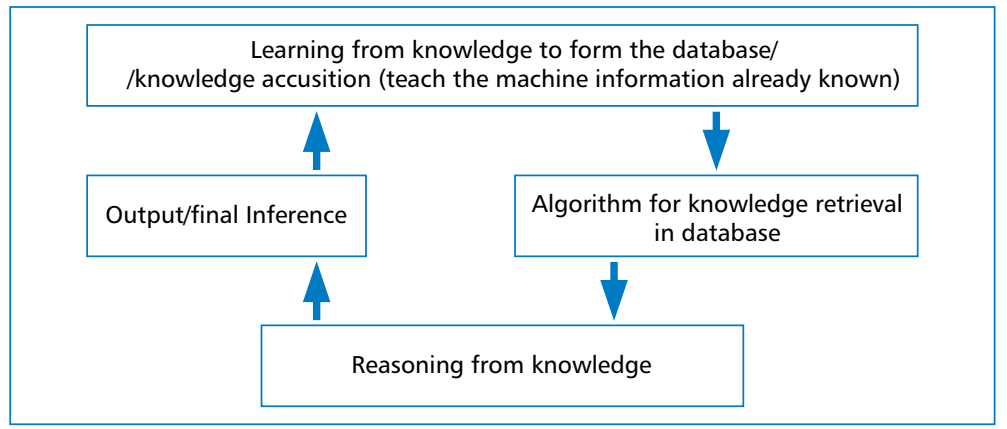

Figure 1. A simplified methodology in understanding the core principle of artificial intelligence

\section{Case-based reasoning}

It is an approach of learning from the past similar encounters to solve the problems of the new problems and this approach is one of the most extensively used in management of diabetes for optimizing and individualization of the insulin therapy for various meals [11, 12]. One of the used CBR systems is the 4-Diabetes support system for automation for mitigating the problem of individual approach of blood glucose management [11].

\section{Machine learning and deep learning}

This method has various learning process which include artificial neural networks (ANNs), support vector machine, naïve Bayes, decision trees, random forest, classification and regression trees, and k-nearest neighbor $[3,4]$. It is used for automated screening for blood glucose variability and helpful in identification of people with diabetes who are at high risk of diabetes due to metabolic of genetic preponderance [12]. The ANNs are being used for diagnosis of diabetes and impact of factors on the glycemic indices for bringing out personalized result for people with diabetes [14].

\section{Miscellaneous}

Techniques like support vector regression has been used to for predictors of hypoglycemia and it help in alerting of the patient when patients have significant hypoglycemia.

The knowledge already known to us first needs to be introduced to the computers to allow the computers to learn automatically without human intervention. It involves various complex convolutional neural networks (e.g. Kalman filter, evolutional algorithms, deep learning etc). After this the algorithms for retrieval of information from the database fed to the computer are formed to make the computer understand which data is important and what is not known as knowledge discovery in database (KDD). Following this, the process of reasoning from the knowledge already acquired results in deduction and inference generation and this final conclusions is added to the database for future reasoning.

\section{Role of Al in management of the diabetic complications}

Hypo- and hyperglycemias

The early recognition and management of the complications (acute as well as chronic) in diabetes predict the QoL for a patient. The promising results of $\mathrm{Al}$ in the early diagnosis of diabetic retinopathy have opened the frontiers for management of other complications as well.

The use of Flash glucose monitors (FGMs) and continuous glucose monitors (CGMs) are recently commercially available and shown promise for effective blood glucose management not only in type 1 but also in insulin requiring type $2 \mathrm{DM}$ [8]. Studies with CGM such as the FreeStyle Libre (Abbott Diabetes care, Alameda, CA,USA) has shown the real time evaluation of glycemic fluctuations can help to better control short-term oscillations and glycometbolic control especially in patients who require multiple insulin injections [15].

CGM has shown to be better performers in comparison to the FGMs in preventing hypoglycaemia. If coupled with an insulin pump, CGM system offers the possibility of closed loop insulin system as commonly known as artificial pancreas, for e.g. Diabeloop technology (Diabeloop SAS, Grenoble, France) which holds promise for type 1 and insulin requiring type $2 \mathrm{DM}$ as well as reducing the total healthcare cost in management of diabetes.

\section{Diabetic retinopathy and macular oedema}

Diabetic retinopathy (DR) is the most serious cause of preventable secondary blindness and annual retinal exam has been advised. It has the limitations of requiring a specialised expert professional and use of mydriatic which limits the activity of the patient for 
the day of examination. Automated diagnosis of diabetic retinopathy and cardiovascular risk factor monitoring are now possible based on the large retinal fundus imaging datasets with sensitivity and specificity over $90 \%[16,17]$. The Food and Drug Administration (FDA) has recently approved the marketing of the medical device called IDx-DR (IDx LLC, Coralville, IA) which uses the software which uses $\mathrm{Al}$ algorithm to analyze the images taken with retinal camera called Topocan NW400 to classify the retinopathy as "more than mild DR" and "negative for more than mild diabetic retinopathy" [5]. The Al algorithm by Medios Technologies, Singapore has shown promise and is the first offline software for DR screening with the smart phone-based fundus camera, the Remidio non-mydriatic fundus-on-phone with sensitivity and specificity of $83.3 \%$ and $95.5 \%$ respectively [18].

\section{Decision support in clinical practice}

Machine learning can be used for prediction of long and short term $\mathrm{HbA} 1 \mathrm{c}$ response at the initiation of insulin in patients with type 2 diabetes. The elastic net regularization based generalized linear model using the baseline $\mathrm{HbA} 1 \mathrm{c}$ and the glomerular filtration rate can help in predicting the short term and long term $\mathrm{HbA1c}$ response [19].

\section{Other uses}

The other uses of Al could be summarized:

- Population risk stratification by the healthcare recommendation system to predict the risk of diabetes. These models are based on large data analytics;

- Genomics- Microbiome data has been used to build the repository of various microbial marker genes which can help in predictability of developing diabetes in future [20];

- Increasing patient's self awareness and treatment - use of mobile applications;

- Remote monitoring of the diabetes status through use of automated real time monitoring devices;

- Life style modification tracking devices-Wearable devices like smart bands and smart scales.

Today Al has future prospects in helping physicians in decision making and to tailor make individual patient's diabetes management and also ensuring adherence to treatment for better health outcomes. Non-invasive diagnosis of diabetes and monitoring the diabetic neuropathy as well as diabetic wounds are being tried with $\mathrm{Al}$ assisted devices and will be commercially available in recent future.

\section{Conclusions}

It is just a beginning of the new era of Al being implemented for health care needs. Al applications offer greater accuracy, efficiency, ease of use, and satisfaction for PWDs, their clinicians, family, and caregivers. The major challenges that Al will be facing is the limitation in reproducing Al from published data, as most of the source code is not publicly available for competitive reasons which demands more transparency with data sharing. Diabetes as a disease has moved from locally recorded few blood glucose and $\mathrm{HbA} 1 \mathrm{c}$ monitoring to development of smart data for the betterment of the patient as well as population of PWD. Smart-phones and smart devices have the potential to bring the management of diabetes to every patient with just a touch on the screen as an "e-disease". Above being said, Al should complement but can never replace what is being done in clinical settings for management of diabetes.

\section{Conflict of interest}

None declared.

\section{REFERENCES}

1. Merriam-Webster. (n.d.). Intelligence. In Merriam-Webster. com dictionary. Retrieved November 11, 2020, from: www. merriam-webster.com/dictionary/intelligence. [Last accessed at: 11.11.2020].

2. Boden MA. Artificial intelligence and natural man. Hassocks, Harvester Press 1977.

3. Ramesh AN, Kambhampati C, Monson JRT, et al. Artificial intelligence in medicine. Ann R Coll Surg Engl. 2004; 86(5): 334-338, doi: 10.1308/147870804290, indexed in Pubmed: 15333167.

4. Ellahham S. Artificial intelligence: the future for diabetes care. Am J Med. 2020; 133(8): 895-900, doi: 10.1016/j.amjmed.2020.03.033, indexed in Pubmed: 32325045.

5. Dankwa-Mullan I, Rivo M, Sepulveda M, et al. Transforming diabetes care through artificial intelligence: the future is here. Popul Health Manag. 2019; 22(3): 229-242, doi: 10.1089/ pop.2018.0129, indexed in Pubmed: 30256722.

6. Thomas RL, Halim S, Gurudas S, et al. IDF diabetes atlas: a review of studies utilising retinal photography on the global prevalence of diabetes related retinopathy between 2015 and 2018. Diabetes Res Clin Pract. 2019; 157: 107840, doi: 10.1016/j. diabres.2019.107840, indexed in Pubmed: 31733978.

7. Karagiannis T, Andreadis $\mathrm{P}$, Manolopoulos A, et al. Decision aids for people with Type 2 diabetes mellitus: an effectiveness rapid review and meta-analysis. Diabet Med. 2019; 36(5): 557-568, doi: 10.1111/dme.13939, indexed in Pubmed: 30791131.

8. Fagherazzi G, Ravaud P. Digital diabetes: Perspectives for diabetes prevention, management and research. Diabetes Metab. 2019; 45(4): 322-329, doi: 10.1016/j.diabet.2018.08.012, indexed in Pubmed: 30243616.

9. McConnell MV, Shcherbina A, Pavlovic A, et al. Feasibility of obtaining measures of lifestyle from a smartphone app: the myheart counts cardiovascular health study. JAMA Cardiol. 2017; 2(1): 67-76, doi: 10.1001/jamacardio.2016.4395, indexed in Pubmed: 27973671.

10. Zhang J, Xu J, Hu X, et al. Diagnostic method of diabetes based on support vector machine and tongue images. Biomed Res Int. 2017; 2017: 7961494, doi: 10.1155/2017/7961494, indexed in Pubmed: 28133611.

11. Marling C, Wiley M, Bunescu R, et al. Emerging applications for intelligent diabetes management. Al Magazine. 2012; 33(2): 67, doi: 10.1609/aimag.v33i2.2410. 
12. Schmidt R, Montani S, Bellazzi R, et al. Cased-Based reasoning for medical knowledge-based systems. Int J Med Inform. 2001; 64(2-3): 355-367, doi: 10.1016/s1386-5056(01)00221-0, indexed in Pubmed: 11734397.

13. Buch V, Varughese G, Maruthappu M. Artificial intelligence in diabetes care. Diabet Med. 2018; 35(4): 495-497, doi: 10.1111/ dme.13587, indexed in Pubmed: 29368355.

14. Odedra D, Samanta S, Vidyarthi AS. Computational intelligence in early diabetes diagnosis: a review. Rev Diabet Stud. 2010; 7(4): 252 262, doi: 10.1900/RDS.2010.7.252, indexed in Pubmed: 21713313.

15. Feig DS, Donovan LE, Corcoy R, et al. Continuous glucose monitoring in pregnant women with type 1 diabetes (CONCEPTT): a multicentre international randomised controlled trial. Lancet. 2017; 390(10110): 2347-2359, doi: 10.1016/S0140-6736(17)32400-5, indexed in Pubmed: 28923465.

16. Gulshan V, Peng L, Coram M, et al. Development and validation of a deep learning algorithm for detection of diabetic retinopathy in retinal fundus photographs. JAMA. 2016; 316(22): 2402-2410, doi: 10.1001/jama.2016.17216, indexed in Pubmed: 27898976.

17. Ting DS, Cheung CYL, Lim G, et al. Development and validation of a deep learning system for diabetic retinopathy and related eye diseases using retinal images from multiethnic populations with diabetes. JAMA. 2017; 318(22): 2211-2223, doi: 10.1001/ jama.2017.18152, indexed in Pubmed: 29234807.

18. Sosale B, Aravind SR, Murthy H, et al. Simple, mobile-based artificial intelligence algoithm in the detection of diabetic retinopathy (SMART) study. BMJ Open Diabetes Res Care. 2020; 8(1), doi: 10.1136/bmjdrc-2019-000892, indexed in Pubmed: 32049632.

19. Nagaraj SB, Sidorenkov G, van Boven J, et al. Predicting short- and long-term glycated haemoglobin response after insulin initiation in patients with type 2 diabetes mellitus using machine-learning algorithms. . Diabetes Obes Metab. 2019; 21(12): 2704-2711.

20. Han W, Ye Y. A repository of microbial marker genes related to human health and diseases for host phenotype prediction using microbiome data. . Pac Symp Biocomput. 2019; 24: 236-247.

21. Rahmani Katigari $M$, Ayatollahi $H$, Malek $M$, et al. Fuzzy expert system for diagnosing diabetic neuropathy. World J Diabetes. 2017; 8(2): 80-88, doi: 10.4239/wjd.v8.i2.80, indexed in Pubmed: 28265346 .

22. Wang L, Pedersen PC, Strong DM, et al. An automatic assessment system of diabetic foot ulcers based on wound area determination, color segmentation, and healing score evaluation. J Diabetes Sci Technol. 2015; 10(2): 421-428, doi: 10.1177/1932296815599004, indexed in Pubmed: 26253144. 